\title{
Strategies for fast and reliable 4D-STEM orientation and phase mapping of nanomaterials and devices
}

\author{
Jian-Min Zuo ${ }^{1}$ and Xiurong $\mathrm{Zhu}^{2}$ \\ ${ }^{1}$ University of Illinois at Urbana-Champaign, Urbana, Illinois, United States, ${ }^{2}$ Emabsoftware Inc, Champaign, \\ Illinois, United States
}

The development of fast electron detectors has significantly improved the speed of acquisition and the field of view in scanning electron nanodiffraction (SEND) or 4D scanning transmission electron microscopy (4D-STEM) for crystallographic orientation and phase imaging. Such imaging requires fast and robust diffraction pattern (DP) indexing and identification of crystal orientation and/or phases. However, a major difficulty is electron dynamical scattering, which renders the diffraction intensity highly dependent on the crystal thickness and orientation. The other difficulty is the large amount of data, where fast analysis is critical. Automatic DP indexing is usually achieved by comparing an experiment DP with a set of simulated DPs and by searching for the best pattern match $[1,2]$ Several algorithms have been reported for spot DP matching. These algorithms can be broadly separated into the intensity and the diffraction vector based approaches [2]. The difficulty with dynamical scattering is removed in the vector-based approach, where indexing is based on the DP geometry alone. However, the use of this method is also limited when the crystal orientation is far away from the zone axis, where there are only a few of diffraction spots. Precession electron diffraction (PED) helps reducing the dynamical effect and increases the number of diffraction spots recorded in a single pattern; both are beneficial for DP indexing[3]. But PED does not render diffraction intensity entirely kinematical, and precession also reduces the sensitivity of diffraction spots to orientation. Using calculated dynamical intensities, for example using the Bloch wave method, could also help with the reliability of DP indexing. But crystal thickness often varies in a nanocrystalline sample, such variations increase the complexity of DP matching and thus the speed of analysis.

To improve indexing reliability, we introduce diffraction spot intensity scaling. The idea of scaling is to reduce the difference between the strong and weak spots in both experimental and simulated patterns, and thus adjust the weights of diffraction intensity in the goodness-of-fit (GOF) parameter used in pattern matching. The scaled intensities $f$ and $g$ are obtained by $F(I, p)$, where $F$ is the scaling function and $p$ for the parameter. We used cross-correlation $\gamma$ for GOF, whose value ranges from -1 to 1 , with 1 for a perfect match. The scaling can be adapted for a variety of DPs. For this option, we define six scaling levels from 0 to 5. At 0 scaling, we take and $\mathrm{f}=\mathrm{I}$ or $\mathrm{g}=\mathrm{I}$, while at the scaling level of 5, we use the thresholding function to reduce diffraction patterns into binary images with the values of 0 or 1 , where 1 is inside a diffraction spot. To avoid interference from the background intensity, we measure the position and intensity of diffraction spots in an experimental DP for subsequent indexing. To generate a simulated DP database, we use the program used in cloudEMAPS for kinematical diffraction pattern simulation [4].

Figure 1 demonstrates the impact of intensity of scaling on two nanobeam diffraction patterns recorded from a nanocrystalline Fe3O4 sample, DP1 is near zone axis (Fig. 1e and f) while the DP2 is off zone axis (Fig. 1g and h). Figures 1a and $\mathrm{c}$ show the GOF maps with scaling level of 1 for the experimental pattern and 2 for the simulated pattern for DP1, while Figures $1 b$ and d show the GOF maps with scaling level of 2 for the experimental pattern and 3 for the simulated pattern for DP2. For DP1, the higher level of scaling reduces the width of peak GOF to a single pixel ( 0.27 degrees wide). The same level scaling, however, leads to multiple maxima and wrong indexing for DP2.

To increase the analysis speed of a 4D dataset, we first separate the DPs into clusters using the image distance method and the obtain the cluster averaged DPs for indexing [5]. By combining the above approaches 
together, we thus have developed an effective strategy for improving the reliability and speed of 4D-STEM orientation and phase mapping analysis, as well as the toolbox for such analysis.
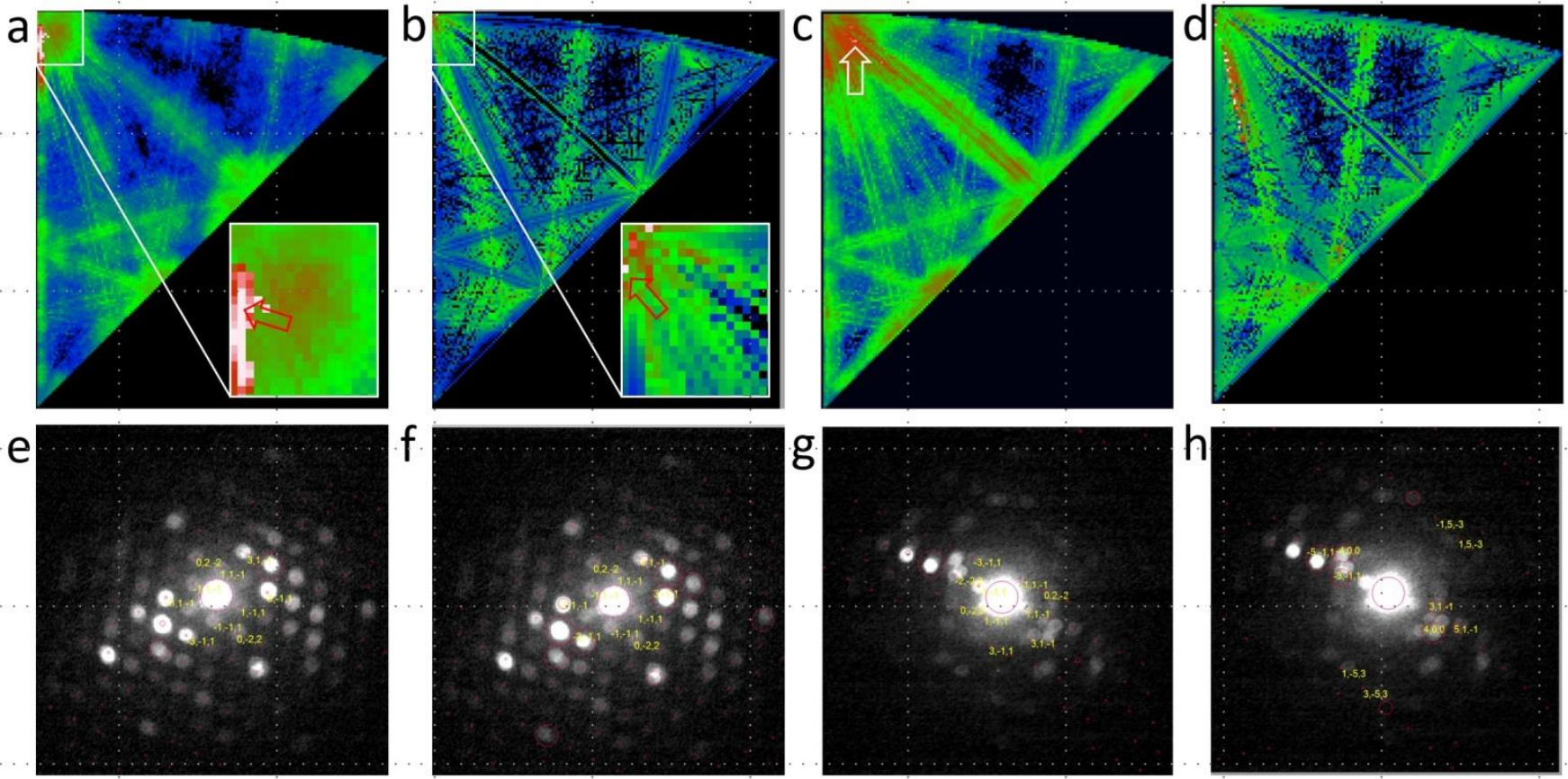

Figure 1. Fig. 1 Diffraction pattern indexing using normalized correlation of scaled experimental and simulated diffraction intensities. Top row, the GOF distribution maps calculated using eq. 1 with two types of intensity scaling. Bottom row are the indexed diffraction patterns with the red circles mark the simulated patterns.

References

[1] E.F. Rauch, A. Duft, Orientation Maps Derived from TEM Diffraction Patterns Collected with an External CCD Camera, Materials Science Forum, 495-497 (2005) 197-202.

[2] Y. Meng, J.-M. Zuo, Improvements in electron diffraction pattern automatic indexing algorithms, Eur. Phys. J. Appl. Phys., 80 (2017) 10701.

[3] P.A. Midgley, A.S. Eggeman, Precession electron diffraction - a topical review, Iucrj, 2 (2015) 126-136.

[4] J.M. Zuo, X.R. Zhu, E. Ang, R. Shah, cloudEMAPS: A Cloud Computing Environment for Electron Microscopy Application Simulations, Microscopy Today, 29 (2021) 24-27.

[5] J.-M. Zuo, Electron nanodiffraction, in: P.W. Hawkes, J.C.H. Spence (Eds.) Springer Handbook of Microscopy, Springer International Publishing, Cham, 2019. 